Latvian Journal of Chemistry, No 1-4, 2010, 152-158

DOI $10.2478 / \mathrm{v} 10161-010-0005-9$

\title{
SYNTHESIS OF 8-HYDROSELENOQUINOLINE AND ITS DERIVATIVES
}

\author{
J. Ashaks, E. Silina, D. Zaruma
}

Institute of Inorganic Chemistry of the Riga Technical University, Miera str. 34, Salaspils, Latvia, e-mail: nki@nki.lv

The methods of synthesis 8-hydroselenoquinoline and its alkyl-, alkyloxy- and phenyl- derivatives have been discussed in the present work. The applicability of potassium selenocyanate and selenourea as the selenium-introducing reagent has been studied. The best results are obtained by the use of selenourea as the selenium-introducing agent. Using this reagent it seems to be possible to obtain some derivatives of 8-hydroselenoquinoline that cannot be synthesized by using potassium selenocyanate as selenium-introducing agent. The corresponding $8,8^{\prime}$ diquinolyldiselenide and methylselenoquinolines synthesized also. The obtained substances characterized by UV-spectra.

Key words: 8-hydroselenoquinoline; 8-hydroselenoquinoline alkylderivatives; 8-hydroselenoquinoline alkyloxy derivatives; 8-hydroselenoquinoline phenyl derivatives.

\section{INTRODUCTION}

8-Hydroselenoquinoline represents itself an analog of the widely known analytical reagents - 8-hydroxyquinoline [1] and 8-mercaptoquinoline [2]. These reagents have found wide application in analytical chemistry for photometric, fluorometric, potentiometric and radiometric determination of minute quantities of heavy metals in the natural and industrial objects as well as for their concentration and separation.

From this point of view, 8-hydroselenoquinoline and its derivatives have been investigated insufficiently. The studies of the analogous reagents containing oxygen, sulfur and selenium offer opportunity to evaluate the influence of the mentioned chalcogens on the physicochemical properties of the reagents themselves and their inner complex compounds. The investigations of interaction of 8-hydroxyquinoline and 8-mercaptoquinoline with metal ions have shown that the latter reagent interacts with the smaller number of metals, that is, it is more selective and its internal complexes are more stable and possess more intense colour. The further improvement of the analytical properties in the case of 8-hydroselenoquinoline is expected.

The latest investigations have shown that 8-hydroselenoquinoline as well as its derivatives and internal complexes possess a definite cytotoxic activity towards the cells of malignant tumors [3-5].

\section{EXPERIMENTAL AND RESULTS}

In the present, 8-hydroselenoquinoline and its derivatives have been obtained: 


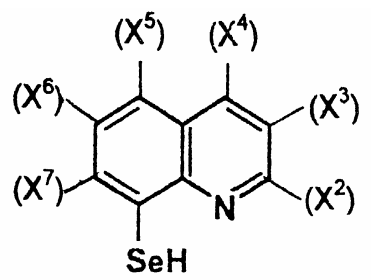

$$
\begin{aligned}
& \mathrm{X}^{2}=\mathrm{CH}_{3} ; \mathrm{C}_{6} \mathrm{H}_{5} ; \\
& \mathrm{X}^{3}=\mathrm{CH}_{3} ; \mathrm{C}_{2} \mathrm{H}_{5} ; \\
& \mathrm{X}^{4}=\mathrm{CH}_{3} ; \mathrm{C}_{2} \mathrm{H}_{5} ; \\
& \mathrm{X}^{5}=\mathrm{CH}_{3} ; \mathrm{OCH}_{3} ; \\
& \mathrm{X}^{6}=\mathrm{CH}_{3} ; \\
& \mathrm{X}^{7}=\mathrm{CH}_{3} ;
\end{aligned}
$$

Such derivatives as 2,4-dimethyl-, 2,5-dimethyl-, 2,6-dimethyl-, 2,7-dimethyl- and 5,6-dimethyl-8-hydroselenoquinolines have been prepared as well.

8-Hydroselenoquinolines have been prepared using two methods in relation to the method of introducing of selenium atom in the quinoline nucleus. The first method provides the introduction of selenium atom in the quinoline molecule using potassium selenocyanate; the second method provides the use of selenourea.

The first method has been applied by Sekido et al. [6, 7]. 8-Hydroselenoquinoline has been synthesized according to the scheme (Fig. 1).<smiles>Nc1cccc2ccc(Br)nc12</smiles>

1<smiles>[N]c1cccc2ccc(C#N)nc12</smiles>

2<smiles>N#Cc1cccc2ccc([Pb])nc12</smiles>

3

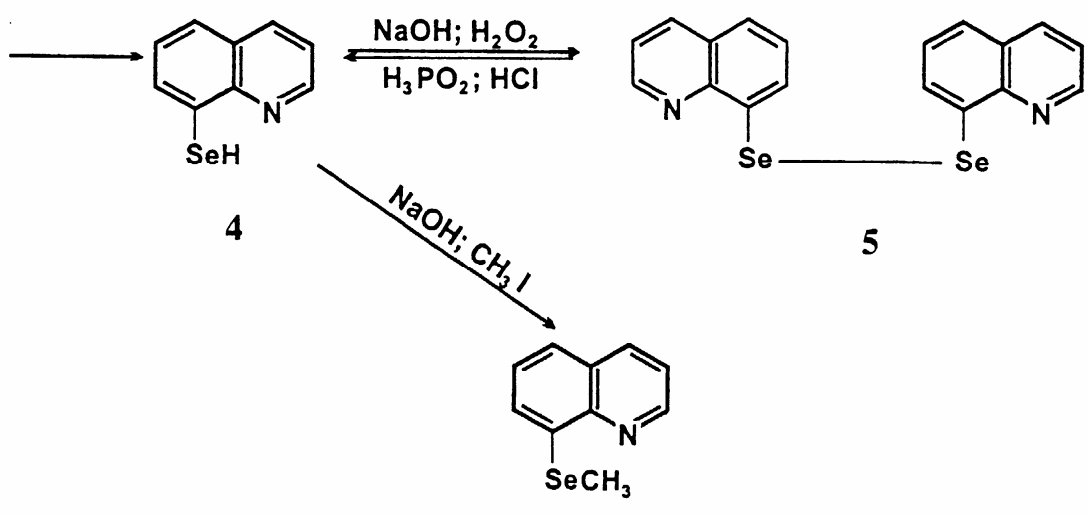

6

Fig. 1. Scheme of synthesis 8 -hydroselenoquinoline by using potassium selenocyanate.

According to this scheme, 8-aminoquinoline (1) is diazotized and by action of potassium selenocyanate on the diazonium salt (2) 8-selenocyanatoquinoline (3) has been obtained. By the acidic hydrolysis of 8-selenocyanatoquinoline (3), 8-hydroselenoquinoline (4) has been prepared. From 8-hydroselenoquinoline, under the action of hydrogen peroxide, 8,8'-diquinolyl diselenide (5) has been obtained, but under the action of methyl iodide in the alkaline medium 8methylselenoquinoline (6) has been prepared.

According to this scheme, Sekido and coworkers have synthesized 8-hydroselenoquinoline [6,7], Bankovsky and coworkers [8] have prepared 2-methyl- 
8-hydroselenoquinoline, but Ashaks and coworkers have obtained 6-methyl[9], 2-phenyl- [10] and 2,4-dimethyl-8-hydroselenoquinoline [11]. Attempts to synthesize 4-methyl-, 7-methyl- and 5-methoxy-8-hydroselenoquinolines in accordance with the above scheme have not been successful, and therefore Ashaks and coworkers have elaborated a new method for the synthesis of 8-hydroselenoquinoline. In this method, selenourea is used as a selenium-introducing reagent instead of potassium selenocyanate. Synthesis of 8-hydroselenoquinoline by this method is represented by the scheme (Fig. 2).
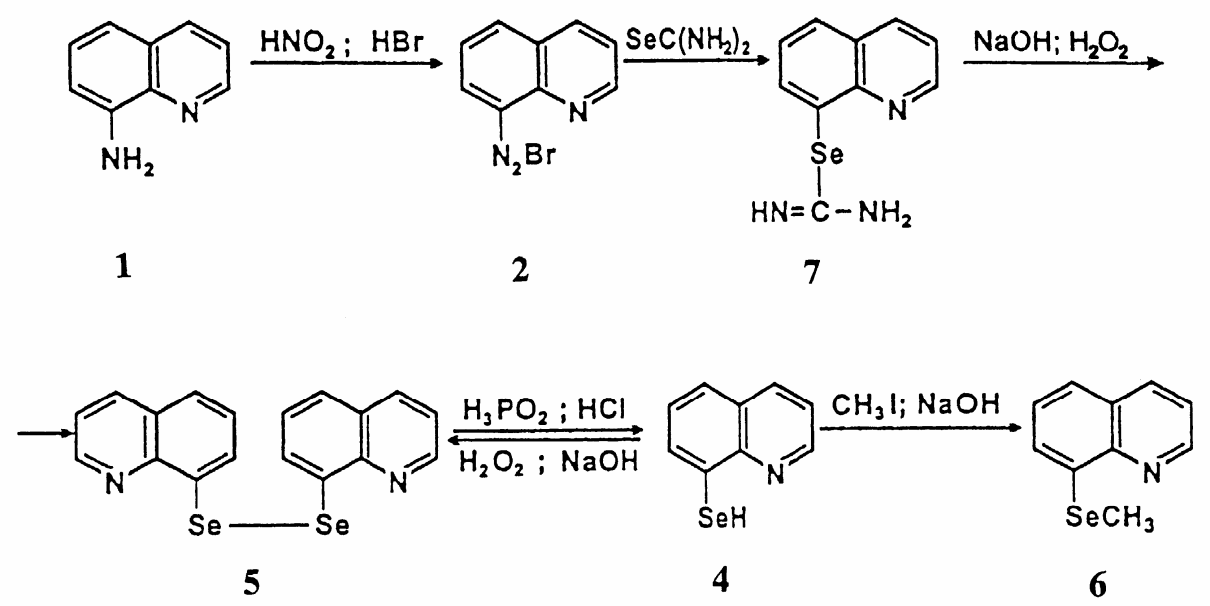

Fig. 2. Scheme of synthesis 8 -hydroselenoquinoline by using selenourea.

8-Aminoquinoline (1) is diazotized and then by the action of selenourea on the obtained diazonium salt (2) quinoline 8-isoselenouronium derivative (7) is obtained. At the alkaline hydrolysis of this isoselenouronium derivative, in the presence of hydrogen peroxide $8,8^{\prime}$-diquinolyl diselenide (5) has been obtained. The latter compound can be reduced by hypophosphorous acid in hydrochloric acidic medium to give 8-hydroselenoquinoline (4) but by the action of methyl iodide on 8-hydroselenoquinoline in the alkaline medium 8-methylselenoquinoline (6) has been prepared.

Using this method, 8-hydroselenoquinoline [12] and its derivatives: 3-methyl[13], 4-methyl- [14], 5-methyl- [15], 7-methyl- [14], 3-ethyl- [16], 4-ethyl- [17], 5-methoxy [18], 2,5-dimethyl- [19], 2,6-dimethyl- [12], 2,7-dimethyl- [19] and 5,6-dimethyl-8-hydroselenoquinolines [20] have been synthesized.

8-Hydroselenoquinolines are red crystalline substances (2-phenyl-8-hydroselenoquinoline is a blue substance), they are easily oxidized by the air oxygen, colourless 8,8'-diquinolyldiselenides (7,7'-dimethyl-8, $8^{\prime}$-diquinolyl diselenide and 2,2',7,7'-tetramethyl-8, $8^{\prime}$-diquinolyl diselenide are yellow) being formed.

8-Hydroselenoquinolines due to their easy oxidability in the air are preserved in the form of the corresponding 8,8'-diquinolyl diselenides, which directly before the use are reduced by hypophosphorous acid in the hydrochloric acid medium during 5-10 min at the ambient temperature.

This yields and melting points of the synthesized compounds are compiled in the Table 1. 
Table 1. Yields and melting points of 8,8'-diquinolyl diselenide, 8-methylselenoquinoline and their derivatives

\begin{tabular}{|c|c|c|c|c|}
\hline Compound & $\begin{array}{l}\text { Method } \\
\text { of syn- } \\
\text { thesis }\end{array}$ & $\begin{array}{c}\text { Yield, } \\
\%\end{array}$ & $\begin{array}{l}\text { Melting } \\
\text { point, }{ }^{\circ} \mathrm{C}\end{array}$ & $\begin{array}{l}\text { Refe- } \\
\text { rence }\end{array}$ \\
\hline $8,8^{\prime}$-Diquinolyl diselenide & 1 & 12 & $205-206$ & 6 \\
\hline 8, $8^{\prime}$-Diquinolyl diselenide & 1 & 40 & 205 & 7 \\
\hline 8, $8^{\prime}$-Diquinolyl diselenide & 2 & 38 & 205 & 12 \\
\hline 2,2'-Dimethyl-8,8'-diquinolyl diselenide & 1 & 11 & $237-238$ & 8 \\
\hline 3,3'-Dimethyl-8,8'-diquinolyl diselenide & 2 & 17 & 263 & 13 \\
\hline 4,4'-Dimethyl-8,8'-diquinolyl diselenide & 2 & 33 & 253 & 14 \\
\hline 5,5'-Dimethyl-8,8'-diquinolyl diselenide & 2 & 28 & $258-260$ & 15 \\
\hline 6,6'-Dimethyl-8,8'-diquinolyl diselenide & 1 & 13 & $231-232$ & 9 \\
\hline 7,7'-Dimethyl-8,8'-diquinolyl diselenide & 2 & 17 & 194 & 14 \\
\hline 3,3'-Diethyl-8,8'-diquinolyl diselenide & 2 & 25 & 207 & 16 \\
\hline 4,4'-Diethyl-8,8'-diquinolyl diselenide & 2 & 30 & 235 & 17 \\
\hline 5,5'-Dimethoxy-8,8'-diquinolyl diselenide & 2 & 34 & $243-244$ & 18 \\
\hline 2,2'-Diphenyl-8,8'-diquinolyl diselenide & 1 & 16 & $247-248$ & 10 \\
\hline 2,2',4,4-Tetramethyl-8, $8^{\prime}$-diquinolyl diselenide & 1 & 14 & $273-274$ & 11 \\
\hline $2,2^{\prime}, 5,5^{\prime}$-Tetramethyl-8, $8^{\prime}$-diquinolyl diselenide & 2 & 27 & 220 & 19 \\
\hline $2,2^{\prime}, 6,6^{\prime}$-Tetramethyl- $8,8^{\prime}$-diquinolyl diselenide & 2 & 24 & 210 & 12 \\
\hline $2,2^{\prime}, 7,7^{\prime}$-Tetramethyl- $8,8^{\prime}$-diquinolyl diselenide & 2 & 15 & 212 & 19 \\
\hline $5,5^{\prime}, 6,6^{\prime}$-Tetramethyl- $8,8^{\prime}$-diquinolyl diselenide & 2 & 27 & $275-277$ & 20 \\
\hline 8-Methylselenoquinoline & 1 & 70 & 60 & 6 \\
\hline 8-Methylselenoquinoline & 2 & 75 & 60 & 12 \\
\hline 2-Methyl-8-methylselenoquinoline & 1 & 75 & $84-85$ & 8 \\
\hline 3-Methyl-8-methylselenoquinoline & 2 & 84 & 92 & 13 \\
\hline 4-Methyl-8-methylselenoquinoline & 2 & 85 & 70 & 14 \\
\hline 5-Methyl-8-methylselenoquinoline & 2 & 84 & 60 & 15 \\
\hline 6-Methyl-8-methylselenoquinoline & 1 & 71 & 74 & 9 \\
\hline 7-Methyl-8-methylselenoquinoline & 2 & 75 & Liquid & 14 \\
\hline 3-Ethyl-8-methylselenoquinoline & 2 & 80 & 70 & 16 \\
\hline 4-Ethyl-8-methylselenoquinoline & 2 & 85 & 83 & 17 \\
\hline 5-Methoxy-8-methylselenoquinoline & 2 & 75 & 116 & 18 \\
\hline 2-Phenyl-8-methylselenoquinoline & 1 & 68 & 71 & 10 \\
\hline 2,4-Dimethyl-8-methylselenoquinoline & 1 & 78 & 108 & 11 \\
\hline 2,5-Dimethyl-8-methylselenoquinoline & 2 & 85 & 52 & 19 \\
\hline 2,6-Dimethyl-8-methylselenoquinoline & 2 & 75 & 101 & 12 \\
\hline 2,7-Dimethyl-8-methylselenoquinoline & 2 & 70 & 35 & 19 \\
\hline 5,6-Dimethyl-8-methylselenoquinoline & 2 & 80 & 71 & 20 \\
\hline
\end{tabular}

Spectral properties of the obtained compounds in the chloroform solutions are presented in the Table 2. 
Table 2. Absorbtion maxima $(\lambda, \mathrm{nm})$ and molar extinction coefficients $(\varepsilon)$ of $8,8^{\prime}$-diquinolyldiselenide, 8 -methylselenoquinoline and their derivatives in chloroform solutions

\begin{tabular}{|c|c|c|c|c|c|}
\hline Compound & $\lambda_{1}, \mathrm{~nm}$ & $\lambda_{2}, \mathrm{nM}$ & $\varepsilon_{1}$ & $\varepsilon_{2}$ & $\begin{array}{l}\text { Refe- } \\
\text { rence }\end{array}$ \\
\hline 8, $8^{\prime}$-Diquinolyl diselenide & 251 & 335 & 38000 & 9600 & 12 \\
\hline 2,2'-Dimethyl-8,8'-diquinolyl diselenide & 254 & 340 & 44000 & 10400 & 8 \\
\hline 3,3'-Dimethyl-8,8'-diquinolyl diselenide & 250 & 325 & 36000 & 11200 & 13 \\
\hline 4,4'- Dimethyl- $8,8^{\prime}$-diquinolyl diselenide & 247 & 334 & 32000 & 12000 & 14 \\
\hline 5,5'- Dimethyl- $8,8^{\prime}$-diquinolyl diselenide & 256 & 347 & 36000 & 10200 & 15 \\
\hline 6,6'-Dimethyl-8,8'-diquinolyl diselenide & 253 & 348 & 44000 & 10800 & 9 \\
\hline 7,7'-Dimethyl-8,8'-diquinolyl diselenide & 245 & 312 & 28000 & 11800 & 14 \\
\hline 3,3'-Diethyl-8,8'-diquinolyl diselenide & 250 & 327 & 36800 & 11200 & 16 \\
\hline 4,4'-Diethyl-8,8'-diquinolyl diselenide & 247 & 335 & 40000 & 12000 & 17 \\
\hline 5,5'-Dimetoxy- $8,8^{\prime}$-diquinolyl diselenide & 260 & 359 & 34000 & 9200 & 18 \\
\hline 2,2'-Diphenyl-8,8'-diquinolyl diselenide & 282 & 347 & 72000 & 17800 & 10 \\
\hline $\begin{array}{l}2,2^{\prime}, 4,4^{\prime} \text {-Tetramethyl- } 8,8^{\prime}- \\
\text { diquinolyl diselenide }\end{array}$ & 253 & 330 & 42000 & 13200 & 11 \\
\hline $\begin{array}{l}2,2^{\prime}, 5,5^{\prime}-\text { Tetramethyl- } 8,8^{\prime}- \\
\text { diquinolyl diselenide }\end{array}$ & 260 & 345 & 44000 & 10800 & 19 \\
\hline $\begin{array}{l}2,2^{\prime}, 6,6^{\prime}-\text { Tetramethyl- } 8,8^{\prime}- \\
\text { diquinolyl diselenide }\end{array}$ & 255 & 341 & 48400 & 10400 & 12 \\
\hline $\begin{array}{l}2,2^{\prime}, 7,7^{\prime} \text {-Tetramethyl- } 8,8^{\prime}- \\
\text { diquinolyl diselenide }\end{array}$ & 315 & 328 & 12400 & 11600 & 19 \\
\hline $\begin{array}{l}\text { 5,5',6,6'-Tetramethyl- } 8,8^{\prime}- \\
\text { diquinolyl diselenide }\end{array}$ & 257 & 350 & 45000 & 10800 & 20 \\
\hline 8-Methylselenoquinoline & 259 & 345 & 16800 & 4600 & 12 \\
\hline 2-Methyl-8-methylselenoquinoline & 260 & 342 & 19600 & 4400 & 8 \\
\hline 3-Methyl-8-methylselenoquinoline & 262 & 343 & 17200 & 5000 & 13 \\
\hline 4-Methyl-8-methylselenoquinoline & 258 & 345 & 16000 & 6000 & 14 \\
\hline 5-Methyl-8-methylselenoquinoline & 263 & 358 & 17000 & 5000 & 15 \\
\hline 6-Methyl-8-methylselenoquinoline & 260 & 347 & 20000 & 5000 & 9 \\
\hline 7-Methyl-8-methylselenoquinoline & 258 & 323 & 14000 & 4000 & 14 \\
\hline 3-Ethyl-8-methylselenoquinoline & 264 & 345 & 17600 & 5000 & 16 \\
\hline 4-Ethyl-8-methylselenoquinoline & 258 & 345 & 16000 & 6200 & 17 \\
\hline 5-Methoxy-8-methylselenoquinoline & 265 & 365 & 15600 & 4300 & 18 \\
\hline 2-Phenyl-8-methylselenoquinoline & 287 & 347 & 33600 & 4800 & 10 \\
\hline 2,4-Dimethyl-8-methylselenoquinoline & 262 & 342 & 19000 & 5800 & 11 \\
\hline 2,5-Dimethyl-8-methylselenoquinoline & 265 & 353 & 20800 & 5000 & 19 \\
\hline 2,6-Dimethyl-8-methylselenoquinoline & 262 & 345 & 22600 & 4500 & 12 \\
\hline 2,7-Dimethyl-8-methylselenoquinoline & 257 & 325 & 12800 & 4400 & 19 \\
\hline 5,6-Dimethyl-8-methylselenoquinoline & 262 & 357 & 20000 & 5000 & 20 \\
\hline
\end{tabular}

\section{CONCLUSIONS}

1. The methods of synthesis of 8-hydroselenoquinoline and its derivatives have been discussed in the present work. The applicability of potassium selenocyanate and selenourea as the selenium-introducing reagents has been studied. 
2. The best results are obtained by the use of selenourea as the seleniumintroducing agent. Using this reagent, it seems to be possible to obtain some derivatives of 8-hydroselenoquinoline that cannot be synthesized using potassium selenocyanate as the selenium-introducing agent.

\section{R E F E R E N C E S}

1. Виноградов, А.В., Элинсон, С.В. (1979). Оксихинолин. Москва. Наука.

2. Банковский, Ю.А. (1987). Химия внутрикомплеесных соединений меркаптохинолина и его производных. Рига. Зинатне.

3. Ашакс, Я., Банковский, Ю., Зарума, Д., Шестакова, И., Домрачева, И., Нестерова, А., Лукевиц, Э. (2004). Синтез 8-хинолинселенола, его комплексных соединений с металлами и их цитотоксическая активность. ХГС, 6, 905-910. [Chem. Heterocycl. Сотр., 40(6), 776-780].

4. Лукевиц, Э., Шестакова, И., Домрачева, И., Нестерова, А., Ашакс, Я., Зарума, Д. (2006). Синтез комплексных соединений метильных производных 8-хинолинселенола с металлами и их цитотоксическая активность. ХГC, 1, 59-66. [Chem. Heterocycl. Сomp., 42(1), 53-59].

5. Лукевиц, Э., Зарума, Д., Ашакс, Я., Шестакова, И., Домрачева, И., Бридане, В., Яценко, Я. (2009). Синтез и цитотоксичность метилзамещенных 8-хинолинселенолатов рутения, родия и иридия. ХГС, 2, 230-236. [Chem. Heterocycl. Comp., 45(2), 182-187].

6. Sekido, E., Fernando, Q., Freiser, H. (1964). An investigation of the dissociation phenomena of quinoline-8-selenol in water and aqueous dioxane. Anal. Chem., 36(9), 1768-1771.

7. Seikido, E., Fujiwara, I. (1972). Improved synthesis and stability of 8-selenoquinoline and its sodium salt as organic reagents. Talanta, 19(5), 647-655.

8. Банковский, Ю.А., Ашакс, Я.В., Зарума, Д.Э. (2002). Синтез, спектры поглощения и константы ионизации 2-метил-8-селенолхинолина. Latvijas Ķimijas Žurnāls, 4, 371-377.

9. Ашакс, Я.В., Банковский, Ю.А., Зарума, Д.Э., Силинь, Э.Я. (2001). Синтез и свойства 6-метил-8-селенолхинолина. Latvijas Ķìmijas Žurnāls, 2, 210-212.

10. Ашакс, Я.В., Банковский, Ю.А., Зарума, Д.Э. (2002). Синтез и свойства 2-фенил-8селенолхинолина. Latvijas Ķìmijas Žurnāls, 4, 434-436.

11. Ашакс, Я.В., Банковский, Ю.А., Зарума, Д.Э. (2001) Синтез и свойства 2,4-диметил-8селенолхинолина. Latvijas Ķìmijas Žurnāls, 3, 311-312.

12. Ashaks, J., Silina, E., Zaruma, D. (2009). Improved method of hydroselenoquinoline and 2,6-dimethyl-8-hydroselenoquinoline synthesis. Latvijas Kìmijas Žurnāls, 4, 353-354.

13. Ashaks, J., Silina, E., Zaruma, D. (2008). Synthesis of 3-methyl-8-hydoselenoquinoline. Latvijas Kìmijas Žurnāls, 1, 103-104.

14. Ашакс, Я., Банковский, Ю., Зарума, Д. (2003). Синтез 4-метил- и 7-метил-8-селенолхинолинатов. Latvijas Ķimijas Žurnāls, 2, 201-202.

15. Ашакс, Я., Зарума, Д., Силинь, Э. (2006). Синтез 5-метил-8-селенолхинолина. Latvijas Ķìmijas Žurnāls, 3, 295-296.

16. Ашакс, Я., Зарума, Д., Силинь, Э.(2005). Синтез 3-этил-8-селенолхинолина. Latvijas Ķìmijas Žurnāls, 2, 197-198.

17. Ашакс, Я., Силинь, Э., Зарума, Д. (2007). Синтез 4-этил-8-селенолхинолина. Latvijas Ķìmijas Žurnāls, 3, 295-296.

18. Ашакс, Я.В., Банковский, Ю.А., Зарума, Д.Э., Силинь, Э.Я. (2002). Синтез и свойства 5-метокси-8-селенолхинолина. Latvijas Ķimijas Žurnāls, 3, 324-325.

19. Ашакс, Я., Зарума, Д., Силинь, Э. (2005). Синтез 2,5-диметил- и 2,7-диметил-8селенолхинолинов. Latvijas Ķīmijas Žurnāls, 1, 85-86.

20. Ashaks, J., Silina, E., Zaruma, D. (2008). Synthesis of 5,6-dimethyl-8-hydroselenoquinoline. Latvijas K̦imijas Žurnāls, 2, 205-206. 


\section{8-SELENOLHINOLĪNA UN TĀ ATVASINĀJUMU SINTĒZE}

J. Ašaks, E. Siliņa, D. Zaruma

K O P S A V I L K U M S

Darbā apskatītas 8-selenolhinolīna un tā atvasinājumu sintēzes metodes, kā selēnu ievadošu reaǵentu izmantojot kālija selenocianātu un selēnurīnvielu. Selēnurīnvielas pielietošana dod labākus rezultātus. Izmantojot šo reaǵentu, izdodas iegūt dažus 8-selenolhinolīna atvasinājumus, kurus ar kālija selenocianāta palīdzību nevar iegūt.

\section{СИНТЕЗ 8-СЕЛЕНОЛХИНОЛИНА И ЕГО ПРОИЗВОДНЫХ}

Я. Ашакс, Э. Силинь, Д. Зарума

P Е 3 Ю M E

В работе рассмотрены методы синтеза 8-селенолхинолина и его производных. Исследовано применение селеноцианата калия и селеномочевины в качестве селеновводящих реагентов. Применение селеномочевины дает лучшие результаты. Применяя этот реагент, удалось получить некоторые производные 8-селенолхинолина, которые с помощью селеноцианата калия синтезировать не удалось. 